\title{
Preparação e caracterização da hexaferrita de bário dopada com niquel e cobalto
}

\section{(Preparation and characterization of nickel and cobalt-doped barium hexaferrite)}

\author{
R. R. Corrêa ${ }^{1}$, C. W. Pachoal ${ }^{2}$ J. L. P. Dominici ${ }^{1}$ \\ ${ }^{1}$ Programa de Pós-Graduação em Engenharia de Materiais, Instituto Federal de Educação, Ciência e \\ Tecnologia do Maranhão, Av. Getúlio Vargas, 04, Monte Castelo, S. Luís, MA, Brasil 65000-000 \\ ${ }^{2}$ Departamento de Física, Universidade Federal do Maranhão,Campus Bacanga, S. Luís, MA, Brasil \\ proncor@yahoo.com.br
}

\begin{abstract}
Resumo
$\mathrm{O}$ objetivo deste trabalho foi a obtenção de ferritas de bário tipo $\mathrm{W}$ com estequiometria $\mathrm{BaCo}_{2-\mathrm{x}} \mathrm{Ni}_{\mathrm{x}} \mathrm{Fe}_{16} \mathrm{O}_{27}$ por meio do método cerâmico tradicional, que é muito difundindo nas indústrias de processamento cerâmico por ser econômico e de relativa facilidade na obtenção de pós-cerâmicos. Materiais aditivados podem afetar as propriedades elétricas, magnéticas e a microestrutura das ferritas por diferentes mecanismos. Assim, temos como objetivo investigar o efeito da substituição dos elementos cobalto e níquel na microestrutura da hexaferrita tipo $\mathrm{W}$ com fórmula estequiométrica $\mathrm{BaCo}_{2-\mathrm{x}} \mathrm{Ni}_{\mathrm{x}} \mathrm{Fe}_{16} \mathrm{O}_{27}, \mathrm{x}=0,0,0,6,1,0$ e 2 .
\end{abstract}

Palavras-chave: hexaferrita, dopagem, Ni, Co.

\begin{abstract}
The purpose of this work was to obtain $W$-type barium ferrites with a $\mathrm{BaCo}{ }_{2-x} \mathrm{Ni}_{x} \mathrm{Fe}_{16} \mathrm{O}_{27}$ composition using the traditional ceramic method commonly used in the ceramic processing industry because it is a cost-effective and relatively easy route for preparing ceramic powders. Doping materials can affect the electrical, magnetic and microstructural properties of ferrites by different mechanisms. Hence, we examined the effect of substituting the elements cobalt and nickel in the microstructure of $W$-type hexaferrite with a stoichiometric composition of $\mathrm{BaCo}_{2-x} \mathrm{Ni}_{x} \mathrm{Fe}_{16} \mathrm{O}_{27}$ with $\mathrm{x}=0.0,0.6,1.0$ and 2.
\end{abstract}

Keywords: hexaferrite, doping, $\mathrm{Ni}$, Co.

\section{INTRODUÇÃO}

Hexaferritas são ferritas que geram o efeito ferrimagnético e que possuem uma estrutura cristalina hexagonal e podem ser obtidas com quantidades estequiométricas de óxidos. As ferritas são classificadas em quatro categorias de acordo com sua estrutura: spinel, garnet, orthoferrites e hexagonal ferrites [1]. A família das hexaferritas hexagonais pode ser classificada com base em suas composições químicas e estrutura cristalina, elas são subdivididas em seis estruturas simples e fundamentais: M, W, Y, X, Z e U. A estrutura destes diferentes tipos de ferritas hexagonais é muito complexa e pode ser considerada como uma composição de blocos $\mathrm{T}, \mathrm{R}$ e $\mathrm{S}$ na direção do eixo-c hexagonal tais como: $\mathrm{SSR} * \mathrm{~S}{ }^{*} \mathrm{~S} * \mathrm{R}$ para o tipo-W e RSR*S* para o tipo-M, onde T é um bloco de quatro camadas de íons oxigênio $\left(\mathrm{O}_{4}-\mathrm{BaO}_{3}-\mathrm{BaO}_{3}-\mathrm{O}_{4}\right)$ com composição $\mathrm{Ba}_{2} \mathrm{Fe}_{8} \mathrm{O}_{14}$, R é um bloco com três camadas de oxigênio $\left(\mathrm{O}_{4}-\mathrm{BaO}_{3}-\mathrm{O}_{4}\right)$ com composição $\mathrm{BaFe}_{6} \mathrm{O}_{11}$ e S (bloco espinélio) é um bloco com dupla camada de oxigênio $\left(\mathrm{O}_{4}-\mathrm{O}_{4}\right)$ com composição $\mathrm{Fe}_{6} \mathrm{O}_{8}$ os termos com asteriscos indicam uma alternância de $180^{\circ}$ em torno do eixo hexagonal [2].

É crescente o estudo das cerâmicas magnéticas por causa do vasto campo de aplicação e a relativa facilidade de processamento, além do baixo custo e de propriedades estruturais que fazem desses materiais excelentes magnetos, que podem ser utilizados nas mais variadas aplicações, tais como ímãs de geladeiras, meios de gravação magnética (fitas cassetes, cartões magnéticos), dispositivos eletromagnéticos (motores elétricos), dispositivos acústicos (alto-falantes), etc.. Aplicações mais promissoras também fazem parte das aplicações desses materiais, assim, estudos avançam para a melhoria de aplicações tais como, uso de nanopartículas magnéticas na física médica, partículas magnéticas porosas no controle de desastres ambientais, proteção contra radiação eletromagnética, capacidade refrigeradora dos materiais magnéticos sob alta pressão (efeito magnetocalórico) [3].

Com o objetivo de modificar algumas propriedades, várias pesquisas são realizadas com o uso de dopantes nas ferritas. As impurezas produzem mudanças nas propriedades elétricas e magnéticas e o conhecimento dessas mudanças nos dá informações dos valores destas propriedades e do limite de aplicação que deve ser usado, assim como o tipo de impureza, o que poderá conduzir a obtenção de ferritas de alta qualidade. Materiais aditivados podem afetar as 
propriedades elétricas, magnéticas e a microestrutura das ferritas por diferentes mecanismos e isto torna as possibilidades de aplicações de tais materiais maiores, uma vez que características de ferritas duras ou moles podem ser obtidas [4]. Esse estudo, com dopagens, é crescente a partir da década de 80 onde foram feitas dopagens substitucionais ao íon $\mathrm{Fe}^{3+}$, possibilitando uma diminuição da variação da magnitude em função da temperatura e da coercividade [5]. As propriedades intrínsecas, como temperatura de Curie, anisotropia magnetocristalina, magnetização de saturação, etc. são diminuídas quando íons $\mathrm{Fe}^{3+}$ são substituídos, como conseqüência as propriedades intrínsecas como a remanência, coercividade, susceptibilidade, também são reduzidas. O que é realizado é a substituição por um íon com maior raio e outro com menor raio, de tal modo que a média entre seus raios se aproxime o máximo do raio do ferro, como é feito com o Co $\left(\mathrm{r}_{\mathrm{Co}}=0,75 \AA\right)$ e titânio $\left(\mathrm{r}_{\mathrm{Ti}}=\right.$ $0,60 \AA)$ o que resulta na média $\left(r_{\mathrm{M}}=0,675 \AA\right)$ que está bem próximo do raio de Fe. Utilizando Co e $\mathrm{Ni}\left(\mathrm{r}_{\mathrm{Ni}}=0,78 \AA\right)$ e o raio médio $\left(\mathrm{r}_{\mathrm{M}}=0,765 \AA\right)$ neste caso a substituição será melhor para o íon $\mathrm{Fe}^{2+}\left(\mathrm{r}_{\mathrm{Fe}}{ }^{2+}=0,87 \AA\right)$ [6-8].

\section{MATERIAIS E MÉTODOS}

A obtenção da hexaferrita $\mathrm{BaCo}_{2-\mathrm{x}} \mathrm{N}_{\mathrm{x}} \mathrm{Fe}_{16} \mathrm{O}_{27}$ foi feita com $\mathrm{BaCO}_{3}, \mathrm{Fe}_{2} \mathrm{O}_{3}, \mathrm{Co}_{3} \mathrm{O}_{4}, \mathrm{e} \mathrm{Ni}_{2} \mathrm{O}_{3}$. Os reagentes foram pesados, levando-se em consideração a estequiometria, e depois colocados em um vaso de polietileno com esferas de aço e postos para moagem por $4 \mathrm{~h}$ em um moinho de bolas, a $150 \mathrm{rpm}$. Em seguida o pó foi calcinado a $1000{ }^{\circ} \mathrm{C}$ por $6 \mathrm{~h}$ em um forno elétrico, depois colocado no moinho de bolas por $2 \mathrm{~h}$. Os pós de $\mathrm{BaCo}_{2-\mathrm{x}} \mathrm{Ni}_{\mathrm{x}} \mathrm{Fe}_{16} \mathrm{O}_{27}$ foram preparados em diferentes porcentagens molares com $\mathrm{x}$ igual a $0,0,6,1,0 \mathrm{e}$ 2. A sinterização nas pastilhas foi feita em forno elétrico a $1300{ }^{\circ} \mathrm{C}$. As pastilhas permaneceram no forno por $4 \mathrm{~h}$. Para a identificação das fases nos pós calcinados de hexaferritas foi utilizado um difratômetro Siemens D500, no intervalo de $2 \theta$ de $20^{\circ}$ a $80^{\circ}$, com radiação Cuk $\alpha$. Para a caracterização microestrutural as amostras foram analisas em um

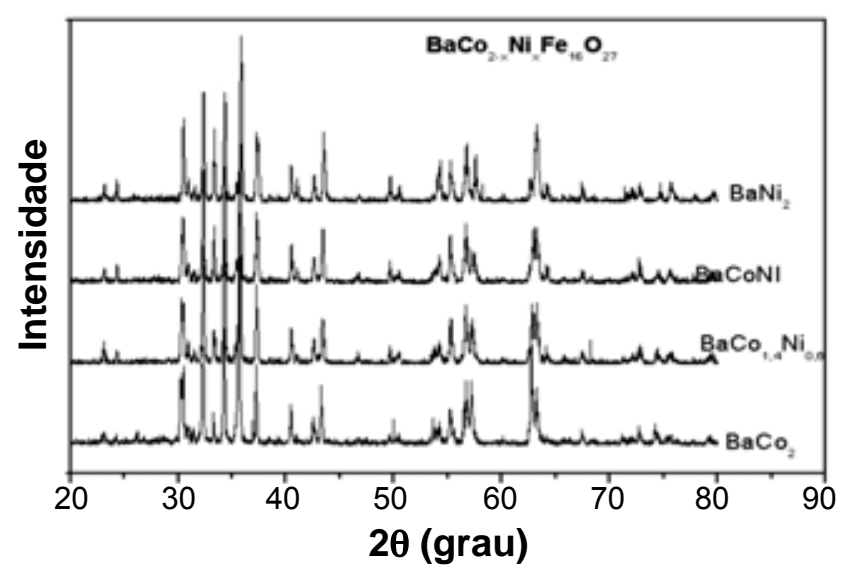

Figura 1: Difratogramas de raios $\mathrm{X}$ das amostras calcinadas a $1000{ }^{\circ} \mathrm{C} / 6 \mathrm{~h}$.

[Figure 1: XRD patterns of samples calcined at $1000^{\circ} \mathrm{C} / 6 \mathrm{~h}$.] microscópio FEG-VP Zeiss Supra 35. As propriedades elétricas em função da freqüência e da temperatura foram determinadas com equipamento Keithley 3330 acoplado a um controlador de temperatura LTC-11, que foi usado para determinar as transições de fase (temperatura de Curie) das cerâmicas.

\section{RESULTADOS E DISCUSSÃO}

A Fig. 1 mostra os difratogramas de raios $\mathrm{X}$ dos pós das quatro amostras calcinadas, podendo-se observar uma semelhança entre os mesmos.

As reflexões basais nos difratogramas de raios $\mathrm{X}$ das amostras foram obtidas no mesmo ângulo de difração. As reflexões obtidas são inerentes à presença da fase hexaferrita; no entanto, a intensidade destas reflexões varia, indicando que esta fase é mais pronunciada em algumas amostras, o que está relacionado à presença dos dopantes. Observase também pela intensidade das reflexões que os dopantes afetam o crescimento dos cristalitos.

A Fig. 2 mostra a relação entre densidade, porosidade e a concentração de níquel, ou seja, tem-se a variação dessas duas grandezas com a quantidade de níquel adicionado a cada composição.

Com o aumento da concentração de níquel, há uma tendência de aumento da porosidade e, em conseqüência, uma diminuição da densidade dos compactos.

Os compactos sinterizados a $1300{ }^{\circ} \mathrm{C}$ por $4 \mathrm{~h}$ foram analisados em microscópio eletrônico de varredura e as micrografias estão apresentadas na Fig. 3. Pode ser observada alta densificação para as amostras sinterizadas nesta temperatura.

A variação no percentual dos elementos dopantes influenciou na forma e no tamanho das partículas e conseqüentemente na distribuição do tamanho das partículas e no grau de aglomeração. As amostras sinterizadas a $1300{ }^{\circ} \mathrm{C}$ também apresentam uma microestrutura com uma distribuição não uniforme de tamanho de grãos e com porosidade maior na composição $\mathrm{BaNi}_{2}$, na qual a distribuição não uniforme do tamanho de grãos é mais acentuada.

A Fig. 4 mostra a relação entre a condutividade dc de uma amostra em função da temperatura. Todas as amostras tiveram comportamento semelhante.

As curvas apresentam duas regiões (inclinações diferentes). Isto indica duas energias de ativação, uma em baixa temperatura e outra em alta temperatura. A mudança na inclinação está associada à mudança no mecanismo de condução. Os baixos valores de energia de ativação em baixas temperaturas estão relacionados à condução extrínseca típica em materiais com dopagem e os altos valores de energia de ativação em alta temperatura estão relacionados a condução intrínseca que passa a dominar após a saturação nos materiais dopados.

A Tabela I mostra os valores das energias de ativação (em baixas e altas temperaturas) em função da composição das amostras, variando entre 0,022 e 0,027 eV (baixa 

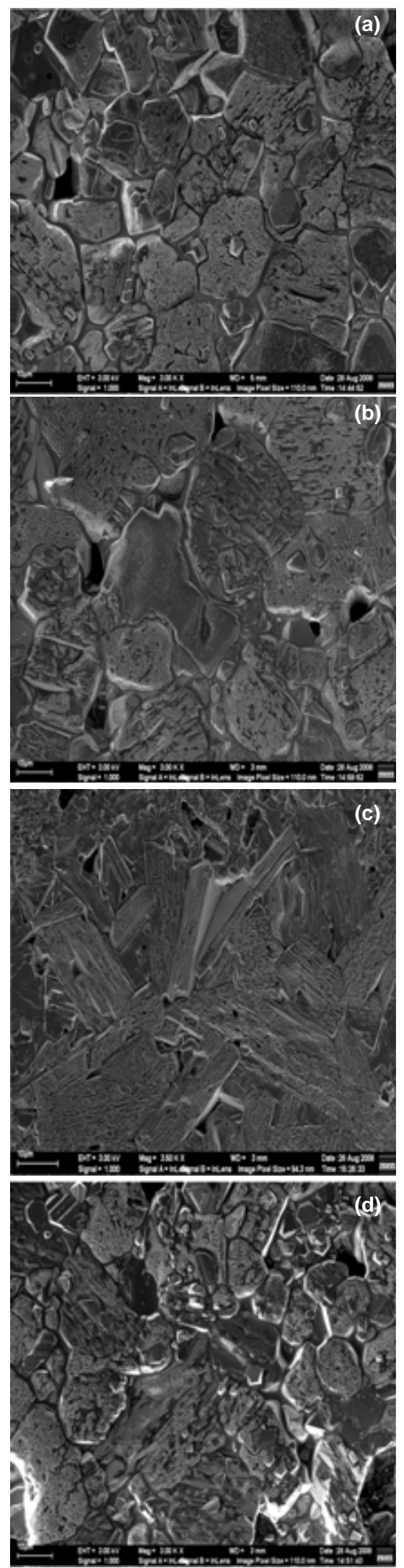

Figura 3: Micrografias obtidas por microscopia eletrônica de varredura dos compactos sinterizados a $1300{ }^{\circ} \mathrm{C} / 4$ h. a) $\mathrm{BaCo}_{2}$, b) $\mathrm{BCo}_{1,4} \mathrm{Ni}_{0,6}$, c) $\mathrm{BaCoNi}$ e d) $\mathrm{BaNi}_{2}$.

[Figure 3: SEM micrographs of the compacts sintered at $1300{ }^{\circ} \mathrm{C} / 4 \mathrm{~h}$. a) $\mathrm{BaCo}_{2}$, b) $\mathrm{BCo}_{1.4} \mathrm{Ni}_{0.6}$ c) $\mathrm{BaCoNi}$ d) $\mathrm{BaNi}_{2}$.]

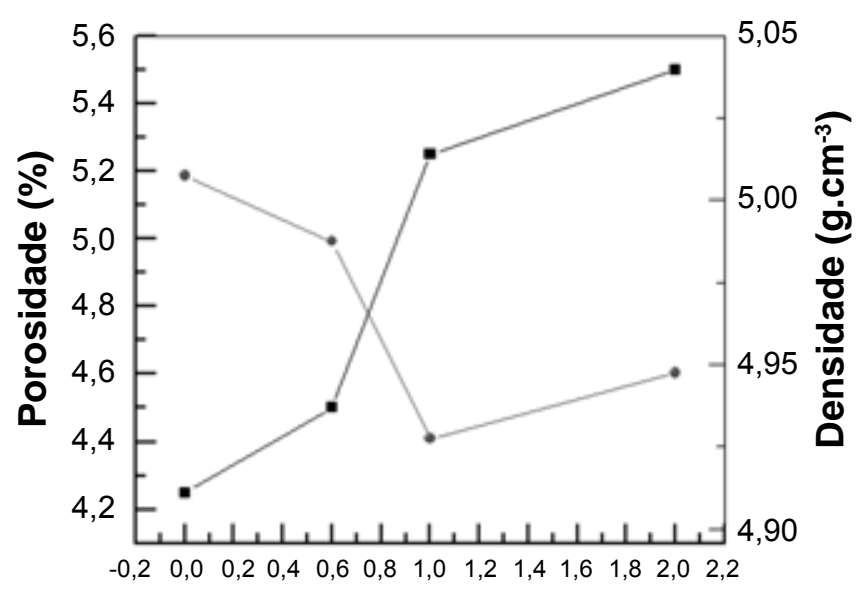

\section{Concentração de níquel}

Figura 2: Relação entre porosidade e densidade para diferentes concentrações de Ni.

[Figure 2: Relationship between the porosity and density for different Ni concentrations.]

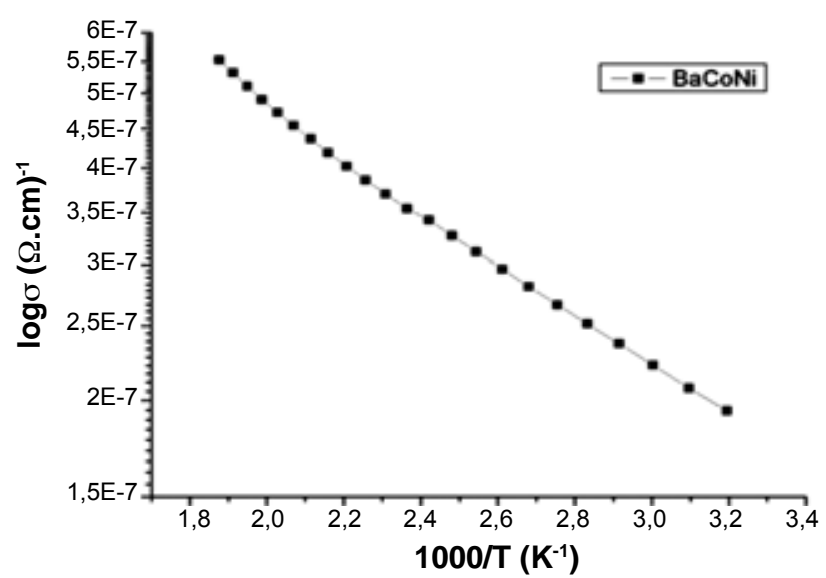

Figura 4: Condutividade elétrica em função da temperatura para BaCoNi calcinado a $1000{ }^{\circ} \mathrm{C} / 6$ h e sinterizado a $1200^{\circ} \mathrm{C} / 4 \mathrm{~h}$, sendo $\mathrm{x}=1,0 \%$ mol Ni na composição $\mathrm{BaCo}_{2-\mathrm{x}} \mathrm{Ni}_{\mathrm{x}} \mathrm{Fe}_{16} \mathrm{O}_{27}$.

[Figure 4: Electrical conductivity versus temperature for the BaCoNi system calcined at $1000^{\circ} \mathrm{C} / 6 \mathrm{~h}$ and sintered at $1200{ }^{\circ} \mathrm{C} / 4$ $h$ where $x=1.0 \mathrm{~mol} \% \mathrm{Ni} \mathrm{BaCo}{ }_{2-x} \mathrm{Ni}_{x} \mathrm{Fe}_{16} \mathrm{O}_{27}$.

Tabela I - Valores de energia de ativação para a condução DC em função da composição $\mathrm{x}$.

[Table I - Values of activation energy for DC conduction as a function of composition $x$.]

\begin{tabular}{ccc}
\hline Composição & \multicolumn{2}{c}{ Energia de Ativação $(\mathrm{eV})$} \\
\hline $\mathrm{X}$ & baixa temperatura & alta temperatura \\
\hline 0 & 0,022 & 0,038 \\
0,6 & 0,029 & 0,032 \\
1 & 0,027 & 0,035 \\
2 & 0,029 & 0,034 \\
\hline
\end{tabular}

temperatura) e 0,032 e $0,038 \mathrm{eV}$ (alta temperatura).

A energia de ativação é mais acentuada em altas temperaturas, sendo que para a composição para $\mathrm{x}=0$, 
contendo apenas cobalto, a energia de ativação é a menor em baixa temperatura e maior em alta temperatura e com a composição para $\mathrm{x}=2$, a energia de ativação é a maior em baixa temperatura.

\section{CONCLUSÕES}

A forma, o tamanho, a distribuição e o grau de aglomeração das partículas foram influenciados pelos elementos dopantes $\mathrm{Co}$ e Ni. No material sinterizado as reflexões basais variaram nas amostras o que está relacionado à presença dos elementos dopantes. A sinterização das pastilhas a $1300{ }^{\circ} \mathrm{C} / 4 \mathrm{~h}$ resultou em um material com uma densidade ideal, sendo a composição $\mathrm{BaCo}_{2} \mathrm{Fe}_{16} \mathrm{O}_{27}$ a que apresentou uma maior densidade média, $5,0 \mathrm{~g} / \mathrm{cm}^{3}$. Houve uma tendência de diminuição de densidade com o aumento do conteúdo de níquel. Os elementos dopantes influenciaram na condutividade elétrica das amostras, nas quais as energias de ativação foram relativamente baixas devido à condução extrínseca e mais elevadas para altas temperaturas devido ao processo de saturação nos materiais dopantes. A composição com $\mathrm{Ba}$ e Co exigiu uma energia de ativação maior em alta temperatura e a composição com $\mathrm{Ba}$ e $\mathrm{Ni}$ exigiu energia de ativação maior em baixa temperatura.

\section{AGRADECIMENTOS}

Ao CNPq, ao IFMA e a UFMA pelo incentivo à pesquisa.

\section{REFERÊNCIAS}

[1] D. El Kony, S. A. Saafan, Study of the temperature dependence of bpth permeability and selectivity of $\mathrm{Mg} \mathrm{Zn}$ hexaferrites, J. Magnetism Magnetic Mater. 267 (2003) 4652.

[2] R. B. Jotania, R. B. Khomane, C. C. Chauhan, S. K. Menon, B. D. Kulkarni, Synthesis and magnetic properties of barium-calcium hexaferrite particles prepared by solgel and microemulsion techniques, J. Magnetism Magnetic Mater. 320 (2008) 1095-1101.

[3] G. Shen, X. Zheng, L. Yi, Absorbing properties and structural design of microwave absorbers based on W-type La-doped ferrite and carbon fiber composites, J. Magnetism Magnetic Mater. 301 (2006) 325-330.

[4] R. Sharma, R. C. Agarwala, V. Argawala, A study on the heat-treatments of nanocrystalline nickel substituted BaW hexaferrite produced by low combustion synthesis method, J. Magnetism Magnetic Mater. 312 (2007) 117-125.

[5] P. S. Sawadh, D. K. Kulkarni, Magnetic and electrical studies $\mathrm{Cu}_{2}-W$ ferrite, Mater. Chem. Phy. 63 (2000) 170 173.

[6] J. Xu G. Hong, Influence of $\mathrm{Nd}^{3+}$ substitution on the microstructure and electromagnetic properties of barium W-type hexaferrite, J. Alloy. Compoun. 490 (2010) 552-556. [7] Vinod N. Dhage, M. L. Mane, A. P. Keche, C. T. Birajdar, K. M. Jadhav, Structural and magnetic behaviour of aluminium doped barium hexaferrite nanoparticles synthesized by solution combustion techniquel, Physica B 406 (2011) 789-793.

[8] P. A. Mariño-Castellanos, A. C. Moreno-Borges, G. Orozsco-Melgar, J. A Garcia, E. Govea-Alcaide, Structural and magnetic study of the $T i^{4+}$-doped barium hexaferrite ceramic samples: Theoretical and experimental results, Physica B 406 (2011) 3130-3136.

(Rec. 16/07/2012, Ac. 18/05/2013) 